DOI: http://doi.org/10.21698/simi.2018.ab35

\title{
MECHANISM OF THE INHIBITION PROCESS IN THE FORMATION OF N-NITROSAMOXICILLIN
}

\author{
Maria Gonta, Elena Sirbu
}

State University of Moldova, Chisinau, MD-2009, 60 Alexei Mateevici, mvgonta@gmail.com, Republic of Moldova

Keywords: $A M X$-amoxicilline, inhibition, NNC-N-nitrosocompounds, quercetine

\section{Introduction}

Among the large number of carcinogens, NNC have an important place, including about $80 \%$ possess carcinogenic action and induce the appearance of malignant tumors in every possible organ of the human body.

Amoxicillin and other antibacterial drugs are secondary amines and possess a free $>\mathrm{N}$ $\mathrm{H}$ group that undergoes the nitrosation process. N-nitrosoamines formed by nitrosation of drugs belong to the class of NNC reagents in which the $>\mathrm{N}-\mathrm{N}=\mathrm{O}$ group is linked to a carbonyl or $>\mathrm{C}=\mathrm{NR}$ group. These compounds, which have mutagenic and carcinogenic effects, belong to the of $\mathrm{N}$-nitrosoamines class, $\mathrm{N}$-nitrosourethanes and $\mathrm{N}$-nitrosoureas. Thus, endogenous nitrosation of drugs in this class leads to the formation of reactive electrophilic compounds, which have an increased toxicity to DNA and can have toxic effects if they are administered at high doses or used for a long time.

\section{Materials and methods}

The kinetics of nitrosation processes were studied following the nitrite consumption and nitrosation substrate. Researches were performed using UV-vis spectrophotometry. The influence of various physicochemical factors on the rate of amoxicillin nitrosation was investigated: variation in nitrate concentration, $\mathrm{pH}$ variation of the reaction medium, and amoxicillin concentration. Studying the process of nitrites transformation according to $\mathrm{pH}$ variation, nitrosation of amoxicillin was studied at $20{ }^{\circ} \mathrm{C}$ in aqueous solutions containing: nitrite concentration of $10^{-4} \mathrm{M}$, amine (amoxicillin) with the concentration of $1 \cdot 10^{-3} \mathrm{M}$, and citrate-phosphate buffer.

\section{Results and conclusions}

The AMX nitrosation process was studied after the $\mathrm{NO}_{2}{ }^{-}$variation using various concentrations of amoxicillin at an acidic $\mathrm{pH}$ of 2.6 which was maintained with the citrate-phosphate buffer solution, the nitrite concentration being $1 \cdot 10^{-4} \mathrm{M}$ and the amine concentrations varied from $1 \cdot 10^{-4} \mathrm{M}$ to $5 \cdot 10^{-3} \mathrm{M}$. Analyzing the experimantal data, we mention that the rate of nitrosation of AMX increases proportionally with its increase in concentration from $1.25 \cdot 10^{-7} \mathrm{M} / \mathrm{s}$ to $4.49 \cdot 10^{-7} \mathrm{M} / \mathrm{s}$ (Figures 1,2 ). Amoxicillin nitrosation was studied also at the rate of the amoxicillin concentration variation (Figures 3, 4). Absorbance in the system after the maximum absorption of the nitrosation substrate according to different parameters was determined. 


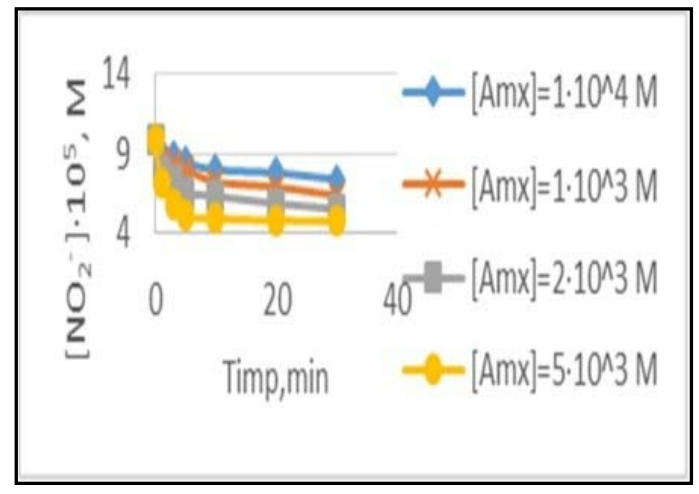

Figure 1. $\left[\mathrm{NO}_{2}^{-}\right]$variation according to $[\mathrm{AMX}] .\left[\mathrm{NO}_{2}{ }^{-}\right]=1 \cdot 10^{-4} \mathrm{M}, \mathrm{pH}=2,6 ; \mathrm{t}=20^{\circ} \mathrm{C}$;

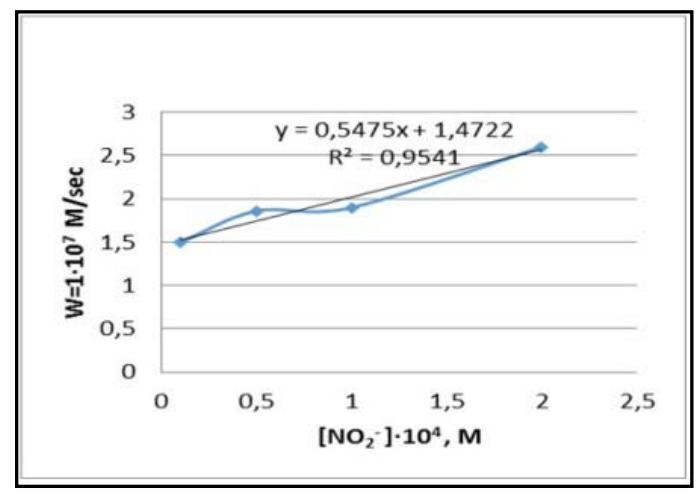

Figure 2. Variation in the consumption rate of nitrite ions according to [AMX]

The rate of AMX nitrosation increases with the increases in AMX concentration, comparing the rate of $\mathrm{AMX}$ nitrosation after $\left[\mathrm{NO}_{2}^{-}\right]$and $[\mathrm{AMX}]$ variation; we find that the first under similar conditions is higher.

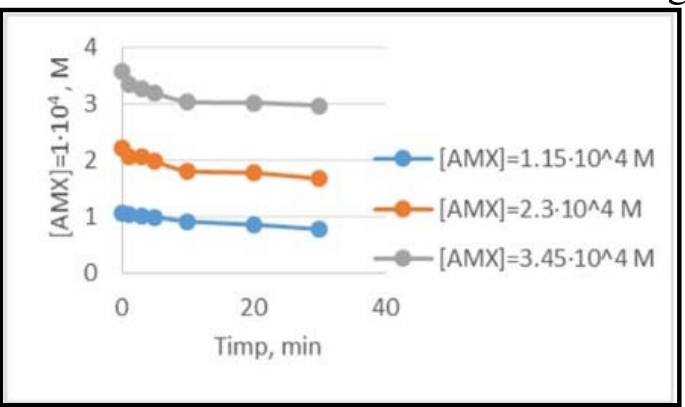

Figure 3. Kinetic curves of variation of $[\mathrm{AMX}]$ to its nitrosation with $\mathrm{NO}_{2}^{-}$.

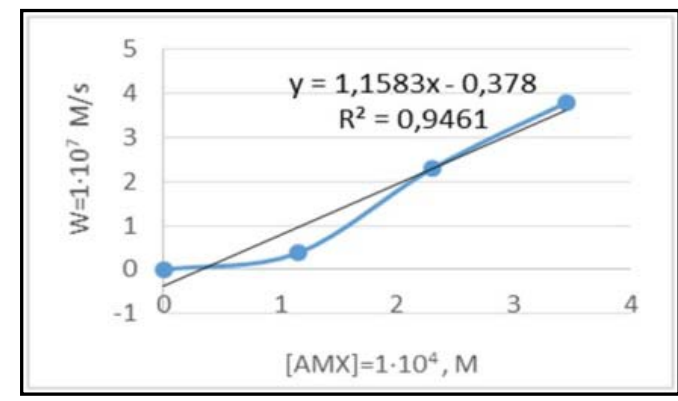

Figure 4. Variation of the rate nitrosation of AMX according to AMX]

It has been established that quercetine and other polyphenols of the flavonoid class have the property of nitrosation processes inhibiting in pharmaceutical products. In order to reduce the AMX nitrosation process, in the experimental investigations it was used different concentrations of quercetine.

In the nitric oxide amoxicillin nitrosation in the presence of quercetine it was found that consumption of $\mathrm{NO}_{2}{ }^{-}$increases with the increase of quercetin concentration and the residual concentration of $\mathrm{NO}_{2}^{-}$in the system decreases. Thus nitrosating agents, which are formed from nitrites, interact not only with AMX but also they interact with the inhibitor, and their concentration in the system decreases further. The higher the quercetin concentration, the higher the rate of consumption of nitrites in the nitrosation process, and the AMX consumption is decreasing. The inhibitory mechanism of the quercetine consists in its ability to nitrate with the nitrosone cation, inhibiting the drug's nitrosation. Studying the amine concentration variation, nitrite nitrosation in the presence of the quercetin reveals that the amine is consumed much less compared to the system lacking the quercetine, which indicates that quercetine has a higher reactivity for the nitrosating agent than AMX, thus AMX concentration decreases.

\section{Acknowledgements}

The researches were carried out within the project STCU 6377. 